\title{
Dysregulation of amino acids and lipids metabolism in schizophrenia with violence
}

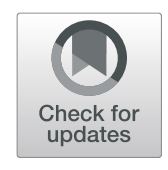

Xiacan Chen ${ }^{1}$, Jiajun $\mathrm{Xu}^{2}$, Jing Tang ${ }^{3}$, Xinhua Dai ${ }^{4}$, Haolan Huang ${ }^{4}$, Ruochen $\mathrm{CaO}^{4}$ and Junmei $\mathrm{Hu}^{4 *}$

\begin{abstract}
Background: Many studies have related biochemical characteristics to violence and have reported schizophrenia could elevated the risk of violent behaviour. However, the metabolic characteristics of schizophrenia patients with violence (V.SC) are unclear.

Methods: To explore the metabolic characteristics of schizophrenia with violence and to identify potential biomarkers, untargeted metabolomics was performed by using gas chromatography time-of-flight mass spectrometry to analyse the plasma metabolites of fifty-three V.SC and twenty-four schizophrenia patients without violence (NV.SC). Multivariate and univariate analyses were performed to identify differential metabolites and biomarkers. Violence was assessed by the MacArthur Violence Assessment Study method. Psychiatric symptoms were assessed by the Positive and Negative Syndrome Scale.

Results: Multivariate analysis was unable to distinguish V.SC from NV.SC. Glycerolipid metabolism and phenylalanine, tyrosine and tryptophan biosynthesis were the differential metabolic pathways between V.SC and NV.SC. We confirmed ten metabolites and five metabolites as metabolic biomarkers of V.SC by random forest and support vector machine analysis, respectively. The biomarker panel, including the ratio of L-asparagine to L-aspartic acid, vanillylmandelic acid and glutaric acid, yielded an area under the receiver operating characteristic curve of 0.808 .

Conclusions: This study gives a holistic view of the metabolic phenotype of schizophrenia with violence which is characterized by the dysregulation of lipids and amino acids. These results might provide information for the aetiological understanding and management of violence in schizophrenia; however, this is a preliminary metabolomics study about schizophrenia with violence, which needs to be repeated in future studies.
\end{abstract}

Keywords: Schizophrenia, Violence, Metabolomics, Biomarker

\section{Background}

Violence is a complex social behaviour and is frequently correlated with schizophrenia [1]. Although only a minority of schizophrenia patients are violent, this violence could lead to a large negative influence on the whole group of patients with schizophrenia. As a result, many studies aim to identify biomarkers of violence among schizophrenia patients to understand the cause of violence and to aid in the prediction and management of violence [2, 3].

In previous studies, the genes of monoamine oxidase A (MAOA) and catechol-O-methyltransferase

\footnotetext{
* Correspondence: junmeihu@hotmail.com

${ }^{4}$ West China School of Basic Medical Sciences \& Forensic Medicine, Sichuan University, Chengdu 610041, China

Full list of author information is available at the end of the article
}

(COMT) genes have been correlated with violence [4, 5]. Abnormalities in the amygdala [6, 7], limbic prefrontal cortex [8,9] and hippocampus [10] have been repeatedly reported to be related to the violent behaviours. Although the neuroimaging findings of aggression and violence were heterogeneous, the structural and functional MRI results have consistently reported abnormalities in the orbitofrontal cortex (OFC) and anterior cingulate cortex (ACC) in schizophrenia [10]. In addition, many findings also suggest the dysfunctions of the cortico-limbic regions, involving the amygdala and prefrontal cortex, in schizophrenia with violence $[3,11]$. In terms of neurotransmitters, the serotonergic system is one of the best studied areas of the neurobiology of violence. Studies have found that 
the relationship between serotonin $(5-\mathrm{HT})$ and violence is complex, but these findings have suggested that 5-HT might influence violence through neural networks involving the amygdala, prefrontal cortex and the striatum [12]. These biomarkers have been applied to some violent behaviour prediction and legal practice [3], but they are hard to be change or regulate directly. It is necessary to explore new regulated biomarkers that could be beneficial to the targeted management of violence.

Metabolites are regulatable factors. Tryptophan is the amino acid precursor of serotonin (5-hydroxytryptamine, 5-HT) biosynthesis [13], and its dietary depletion can reduce central 5-HT concentration [14]. Studies have found that decreased plasma tryptophan levels are related to a higher rate of aggressive behaviours $[15,16]$. Moreover, Thurmond reported higher tyrosine increased aggressive behaviours in animal experiments [17]. Earlier studies reported glycine mediates the defend reaction types in mouse behaviours [18]. In addition, low levels of cholesterol associated with aggression are frequently reported [19-22]. Recently, many studies have found dietary omega-3 supplementation can reduce violent behaviours in children, young men and schizophrenia patients [23-28].

Limited by techniques, many studies mainly focus on one or two metabolites; therefore, a holistic view of the metabolic phenotype of violence might provide more information to help us understand violence. Metabolomics is able to provide a holistic view. It has been applied to the diagnosis, mechanism of disease, the identification of new drug targets and the monitoring of treatment outcomes [29, 30]. To find diagnostic biomarkers of schizophrenia, the metabolomics studies were conducted in different samples, such as peripheral blood mononuclear cells [31], serum [32], plasma [33] and urine [34]. Furthermore, the signatures reported by metabolomics studies are in agreement with the polyunsaturated fatty acid metabolism hypothesis [35] and the membrane phospholipid hypothesis [36] of schizophrenia [37]. A study has validated the potential of tryptophan to be a biomarker for aggression [38]. Another study suggested using lipid levels to predict violent behaviours in schizophrenia [22]. Furthermore, untargeted metabolomics is a method that is independent of priori assumptions, which could provide a holistic approach to understanding the phenotype [39]. To our knowledge, studies have reported metabolome studies of violence in animal models [40-42], but none in schizophrenia patients. Consequently, we speculate that schizophrenia patients with violence (V.SC) might differ from schizophrenia patients without violence (NV.SC) in metabolic phenotype.
We hypothesized that V.SC would present metabolic characteristics that differ from those of NV.SC. Therefore, in this study, we compared the metabolic biomarkers of V.SC with those of NV.SC by using untargeted metabolomics and profiled the metabolic status of V.SC in a holistic perspective. This study also conducted a feature selection analysis to further explore the predictive utility of discovered metabolic biomarkers in discriminating schizophrenia patients with violence from those without violence.

\section{Methods}

Patients were recruited from the inpatient department of Chengdu Compulsory Medical Center (25 samples) and Chengdu Jinxin Mental Health Center (52 samples), Sichuan, China. They were all aged $18-40$ years, of Han nationality and lived in Sichuan in the last year. They were screened by psychiatric and forensic psychiatric graduate students using the Structured Clinical Interview for DSM-IV. Patients were ultimately diagnosed with schizophrenia by two senior psychiatrists according to DSM-IV criteria and the interview. They were naïve to using any antibiotics in the last 3 months. Patients were excluded if they had been diagnosed with hypertension, diabetes, cirrhosis, immunodeficiency, autoimmune diseases and cancer in the last 3 months. Patients with organic mental disorder, mental and behavioural disorders due to psychoactive substance use, mental retardation and alcohol/drug abuse or dependence were also excluded. Pregnant or breastfeeding women were excluded. Patients with a history of suicide/self-injury were excluded. The immediate families of all participants had signed the informed consent. The ethics approval of this study was approved by the Medical Ethics Committee of Sichuan University.

Patients were classified into two groups-schizophrenia patients with violence (V.SC) and schizophrenia patients without violence (NV.SC). Violent behaviour was identified according to the serious violence classification in the MacArthur Violence Risk Assessment Study (MVRAS): 1) batteries that resulted in physical injury or involved the use of a weapon, 2) sexual assaults, and 3) threats made with a weapon in hand [43]. If the patients acted any of the above behaviours since they were diagnosed with schizophrenia, they were assigned to the V.SC group. Patients who never acted any of the above behaviours were assigned to the NV.SC group.

\section{Psychiatric symptom assessment}

A questionnaire was conducted to collect demographic information. The Positive and Negative Syndrome Scale (PANSS) was used by three psychiatric and forensic psychiatric graduate students to assess psychotic symptoms $(\mathrm{ICC}=0.983)$. 


\section{Untargeted metabolomics analysis}

Fasting venous blood specimens were collected from 77 participants in EDTA anticoagulant tubes between 7:00 and 7:30 a.m.. The fresh blood samples were transported to the laboratory in cold chain $\left(4{ }^{\circ} \mathrm{C}\right)$ in $20 \mathrm{~min}$ and then centrifuged at $1000 \mathrm{~g}, 4^{\circ} \mathrm{C}$ to obtain plasma. The plasma was put in a liquid nitrogen tank for cold extraction for $15 \mathrm{~min}$ and then put in a$80^{\circ} \mathrm{C}$ freezer until analysis.

\section{Plasma sample preparation for metabolomics}

To separate the debris or lipid layer, thawed plasma samples were centrifuged at $3000 \mathrm{~g}, 4{ }^{\circ} \mathrm{C}$ for $5 \mathrm{~min}$. For each sample, $50 \mu \mathrm{l}$ of plasma was mixed with $10 \mu \mathrm{l}$ of internal standard, and $175 \mu \mathrm{l}$ of pre-cooled methanol/chloroform $(\mathrm{v} / \mathrm{v}=3 / 1)$ was added. The mixture was frozen at $-20^{\circ} \mathrm{C}$ for $20 \mathrm{~min}$ and centrifuged at $14,000 \mathrm{~g}, 4^{\circ} \mathrm{C}$ for $4 \mathrm{~min}$. For all samples, the supernatant was concentrated to near dryness using a Centrivap vacuum concentrator (Labconco, Kansas City, MO, USA) to remove chloroform and was then further lyophilized using a freeze dryer (Labconco, Kansas City, MO, USA). Then, $50 \mu \mathrm{l}$ of oximation reagent (a pyridine solution of methoxyamine hydrochloride, $20 \mathrm{mg} / \mathrm{ml}$ ) was added to the dried sample, and incubated at $30^{\circ} \mathrm{C}$ for $2 \mathrm{~h}$. Then, $50 \mu \mathrm{l}$ of silylation reagent (MSTFA+ with FAME) in 1\% TMCS) was added to each sample, which was silanized for 1 $\mathrm{h}$ at $37.5^{\circ} \mathrm{C}$ using an automatic injector. The derivatized sample was then injected into the GC-TOF/MS analyser using an automatic injector. The derivatization and injection of the above samples were performed by the MPS2 Multi-Function Autosampler.

\section{GC-TOF/MS analysis}

Each $1 \mu \mathrm{l}$ derived sample was injected into gas chromatography time-of-flight mass spectrometer (GC-TOF/ MS) system (Pegasus HT, Leco Corp., St. Joseph, MI, USA), with helium as the carrier gas at a flow rate of 1.0 $\mathrm{ml} / \mathrm{min}$. An Rxi-5 ms capillary column $(30 \mathrm{~m} \times 250 \mu \mathrm{m}$ i.d., $\quad 0.25-\mu \mathrm{m}$ film thickness; Restek corporation, Bellefonte, PA, USA) was used for metabolite separation. The injection temperature was set at $270^{\circ} \mathrm{C}$. The oven temperature programming was set as follows: maintaining $80^{\circ} \mathrm{C}$ for $2 \mathrm{~min}$, then raising to $300^{\circ} \mathrm{C}$ at a rate of $12^{\circ} \mathrm{C} / \mathrm{min}$, maintaining $300^{\circ} \mathrm{C}$ for $4.5 \mathrm{~min}$, then raising to 320 at a rate of $40^{\circ} \mathrm{C} / \mathrm{min}$, and maintaining $320^{\circ} \mathrm{C}$ for $1 \mathrm{~min}$. The transfer interface and ion source temperature were set as $270{ }^{\circ} \mathrm{C}$ and $220^{\circ} \mathrm{C}$, respectively. The mass spectrometer adopted an electron impact ionization mode of $-70 \mathrm{eV}$, a detector voltage of $-1450 \mathrm{~V}$, and an acquisition rate of 25 spectra/sec, in a full scan mode ranging from 50 to $550 \mathrm{amu}$.

\section{Metabolomics data analysis}

The retention index and mass spectrometry data were compared with the previous JiaLib ${ }^{\text {mi }}$ metabolite database to complete the metabolite identification by using Xplore MET software (v3.0, Metabo-Profile, Shanghai, China) [44]. More details about the JiaLib ${ }^{\text {Tw }}$ metabolite database, Xplore MET software, raw mass spectral data processing and data preprocessing are provided in the Supplement. Principal component analysis (PCA) was used to observe the trend of aggregation in group and the trend of separation between groups. Orthogonal partial least square discriminant analysis (OPLS-DA) were used to discriminate V.SC patients from NV.SC patients. PCA is an unsupervised modelling method that is commonly used to detect data outliers, clustering, and classification trends without prior knowledge of the sample set [45]. OPLS-DA has been widely used for multi-class classification and the identification of different changing metabolites [46, 47].

The Wilcoxon-Mann-Whitney rank sum test was used to select plasma metabolites that were significantly differed between V.SC and NV.SC $(P>0.05)$. Random forest (RF) analysis applies an ensemble technique by using bootstrap resampling technology and is an effective method for classification and feature selection $[48,49]$. The support vector machine (SVM) is a machine learning classifier and can mitigate the effects of noisy data [50]. Both have been widely been employed for biomarker discovery [51, 52]. RF and SVM analyses were separately used to evaluate the importance of the differential plasma metabolites identified by the Mann-Whitney-Wilcoxon rank sum test and to select plasma metabolic biomarkers. RF analysis adopted Boruta algorithm (maxRun $=1000$ ) by using Boruta package of $\mathrm{R}$ studio. Only if the $p<0.01$, the feature can be identified as "confirmed" and the Bonferroni post-hoc correction was used in $p$ value of results [48]. SVM analysis adopted 7 fold cross validation and repeated 100 times by using svm () function in R package e1071 [53]. Only the plasma metabolites that meet both RF (marked as "confirmed") and SVM (importance >60, or the number of metabolites with importance above $60<5$, the five most important metabolites) feature selection criterion can be identified as metabolic biomarkers and selected to form final biomarker panel. Receiver operating characteristic (ROC) curve was used to assess the validity of plasma metabolic biomarkers in discriminate V.SC from NV.SC.

Metabolic pathway enrichment analysis (MPEA) is a commonly used method for metabolic pathway analysis. MPEA can help detect biologically meaningful metabolite sets that have been enriched in human metabolomic studies [54]. We used MPEA to identify differential 
metabolic pathways and illustrate meaningful metabolites in these ways.

\section{Results}

\section{Clinical characteristics}

The V.SC group consisted of 53 patients and the NV.SC group consisted of 24 patients. The duration of inpatients ranged from 0.2 to 14 years (V.SC: $0.3-14.0$ years; NV.SC: $0.2-8.0$ years) and differed significantly between groups. There was no statistically significant difference between the two groups in terms of other demographic characteristics and clinical symptoms. Risperidone and clozapine were the most frequently used antipsychotic drugs. In the V.SC group, 51 patients (96.2\%) had batteries resulting in physical injury, 32 patients (60.4\%) had batteries involving the use of a weapon, 9 patients (17.0\%) made threats with a weapon in hand, and no patient had sexual assaults, after they were diagnosed with schizophrenia (Table 1).

\section{Plasma metabolic profiles of schizophrenia with violence} A total of 236 plasma metabolites were detected by GCTOF/MS analysis, among which 129 were annotated by JiaLib $^{\mathrm{TM}}$ metabolite database.

\section{Discrimination model}

The PCA shows no separation trend between the V.SC and NV.SC groups (Supple. Figure 1 in Supplement).

Table 1 Comparison of the demographic characteristics, clinical symptoms and violence situation between the V.SC and NV.SC groups

\begin{tabular}{|c|c|c|c|c|c|c|}
\hline & $\begin{array}{l}\text { NV.SC } \\
N=24\end{array}$ & & $\begin{array}{l}\text { V.SC } \\
N=5\end{array}$ & & $t / x^{2}$ & $P$ \\
\hline Age (Mean, SD) & 30.9 & 5.2 & 32.6 & 4.7 & -1.45 & 0.15 \\
\hline Male $(N, \%)$ & 13 & 54.2 & 38 & 71.7 & 2.27 & 0.13 \\
\hline \multicolumn{7}{|l|}{ Marriage status (N, \%) } \\
\hline Married & 6 & 25.0 & 6 & 11.3 & \multirow[t]{3}{*}{2.91} & \multirow[t]{3}{*}{0.23} \\
\hline Divorce & 2 & 8.3 & 9 & 17.0 & & \\
\hline Alone & 16 & 66.7 & 38 & 771.7 & & \\
\hline BMI (Mean, SD) & 23.0 & 4.8 & 22.8 & 3.0 & 0.13 & 0.90 \\
\hline Educational years (Mean, SD) & 10.3 & 4.4 & 9.7 & 3.9 & 0.60 & 0.55 \\
\hline \multicolumn{7}{|l|}{ Occupation(N, \%) } \\
\hline Other jobs & 5 & 20.8 & 5 & 9.4 & \multirow[t]{3}{*}{1.98} & \multirow[t]{3}{*}{0.37} \\
\hline Peasantry & 2 & 8.3 & 4 & 7.5 & & \\
\hline Unemployed & 17 & 70.8 & 44 & 83.0 & & \\
\hline Smoking $(N, \%)$ & 10 & 41.7 & 29 & 54.7 & 1.13 & 0.29 \\
\hline First-episode SC. (N, \%) & 3 & 12.5 & 2 & 3.8 & 2.08 & 0.15 \\
\hline Duration of SC. (Mean, SD, year) & 9.1 & 4.0 & 9.5 & 5.4 & -0.34 & 0.74 \\
\hline Duration of inpatient (Mean, SD, year) & 1.7 & 2.3 & 3.7 & 3.7 & -2.41 & 0.02 \\
\hline Duration of antipsychotic treatment (Mean, SD, year) & 8.0 & 4.7 & 8.6 & 5.4 & -0.46 & 0.64 \\
\hline Risperidone (N, \%) & 11 & 45.8 & 23 & 43.4 & 0.04 & 0.84 \\
\hline Clozapine $(N, \%)$ & 10 & 41.7 & 30 & 56.6 & 1.48 & 0.22 \\
\hline \multicolumn{7}{|l|}{ Violent behaviours during SZ. $(N, \%)$} \\
\hline Batteries resulting in physical injury & 0 & 0 & 51 & 96.2 & 68.40 & 0.00 \\
\hline Batteries involving the use of a weapon & 0 & 0 & 32 & 60.4 & 24.80 & 0.00 \\
\hline Sexual assaults & 0 & 0 & 0 & 0 & / & / \\
\hline Threats made with a weapon in hand & 0 & 0 & 9 & 17.0 & 4.62 & 0.03 \\
\hline \multicolumn{7}{|l|}{ PANSS (Mean, SD) } \\
\hline Positive symptom & 11.5 & 7.0 & 10.7 & 4.8 & 0.61 & 0.54 \\
\hline Negative symptom & 16.0 & 8.6 & 15.7 & 8.0 & 0.17 & 0.87 \\
\hline General psychotic symptom & 27.2 & 9.0 & 24.9 & 5.3 & 1.42 & 0.16 \\
\hline PANSS total score & 54.8 & 19.7 & 50.8 & 12.6 & 1.04 & 0.30 \\
\hline
\end{tabular}


The OPLS-DA model shows poor predictive utility in discriminating V.SC group and NV.SC group $\left(R^{2} Y=\right.$ 0.669, $\left.Q^{2}=0.112\right)$ (Fig. 1).

\section{Group differences}

In univariate analysis, nineteen plasma metabolites differed significantly between the V.SC group and NV.SC group $(P<0.05)$, including seven organic acids, five amino acids, four carbohydrates, one lipid, one aldehyde and one alcohol. Three metabolites increased in V.SC, and their fold change (FC) of them ranged above 1.2. Sixteen metabolites decreased in V.SC, and the FC of twelve metabolites ranged below 0.83 . The nineteen differential metabolites mainly participated in amino acid metabolism, lipid metabolism and carbohydrate metabolism (Fig. 2a and Table 2).

\section{Biomarker panel selection}

\section{Assessment of importance}

We used RF analysis to assess the importance (Imp) of the nineteen differential metabolites and nine metabolites were excluded. Ten metabolites were confirmed as metabolic biomarkers of the V.SC group by RF analysis (Imp> 2.5) (Fig. 2b), including D-ribose, 3-aminoisobutanoic acid, glycerol 3-phosphate, ratio of L-asparagine to L-aspartic acid, glutaric acid, ribitol, vanillylmandelic acid, glyceraldehyde, 3-aminosalicylic acid and 4-hydroxyproline.

We also used RF analysis to assess the importance of the nineteen differential metabolites. We selected the five most important metabolites as metabolic biomarkers of the V.SC group, including L-methionine, the ratio of L-asparagine to L-aspartic acid, glutaric acid, L-sorbose and vanillylmandelic acid (Fig. 2c).

To avoid over-fitting issue, only the plasma metabolites that meet both RF and SVM feature selection criterion can be identified as metabolic biomarkers and selected to form final biomarker panel to discriminate the V.SC group from the NV.SC group. Three plasma metabolites meet the criterion, including ratio of $\mathrm{L}$ asparagine to L-aspartic acid, vanillylmandelic acid and glutaric acid. The biomarker panel formed by the three metabolites, yielded an area under the receiver operating characteristic curve (AUC) of 0.808 (Fig. 2d).

\section{Differential metabolic pathways}

Two metabolic pathways, glycerolipid metabolism and phenylalanine, tyrosine and tryptophan biosynthesis were identified as significantly differential pathways between the V.SC and NV.SC groups by MPEA (Fig. 3a and Table 3). In the glycerolipid metabolism pathway, glycerol was significantly up-regulated and glycerol 3-

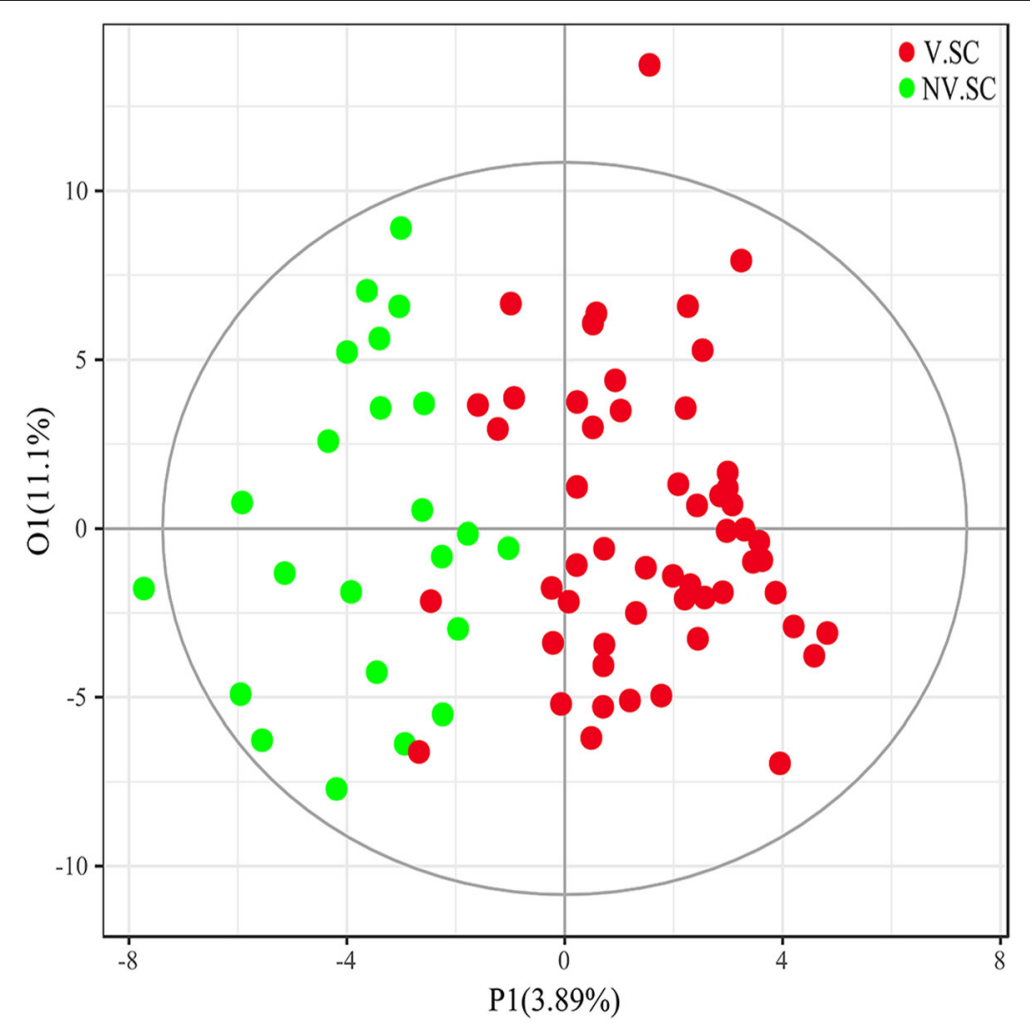

Fig. 1 Orthogonal partial least square discriminant analysis (OPLS-DA) was used to discriminate the V.SC group from the NV.SC group. V.SC: schizophrenia patients with violence; NV.SC: schizophrenia patients without violence 


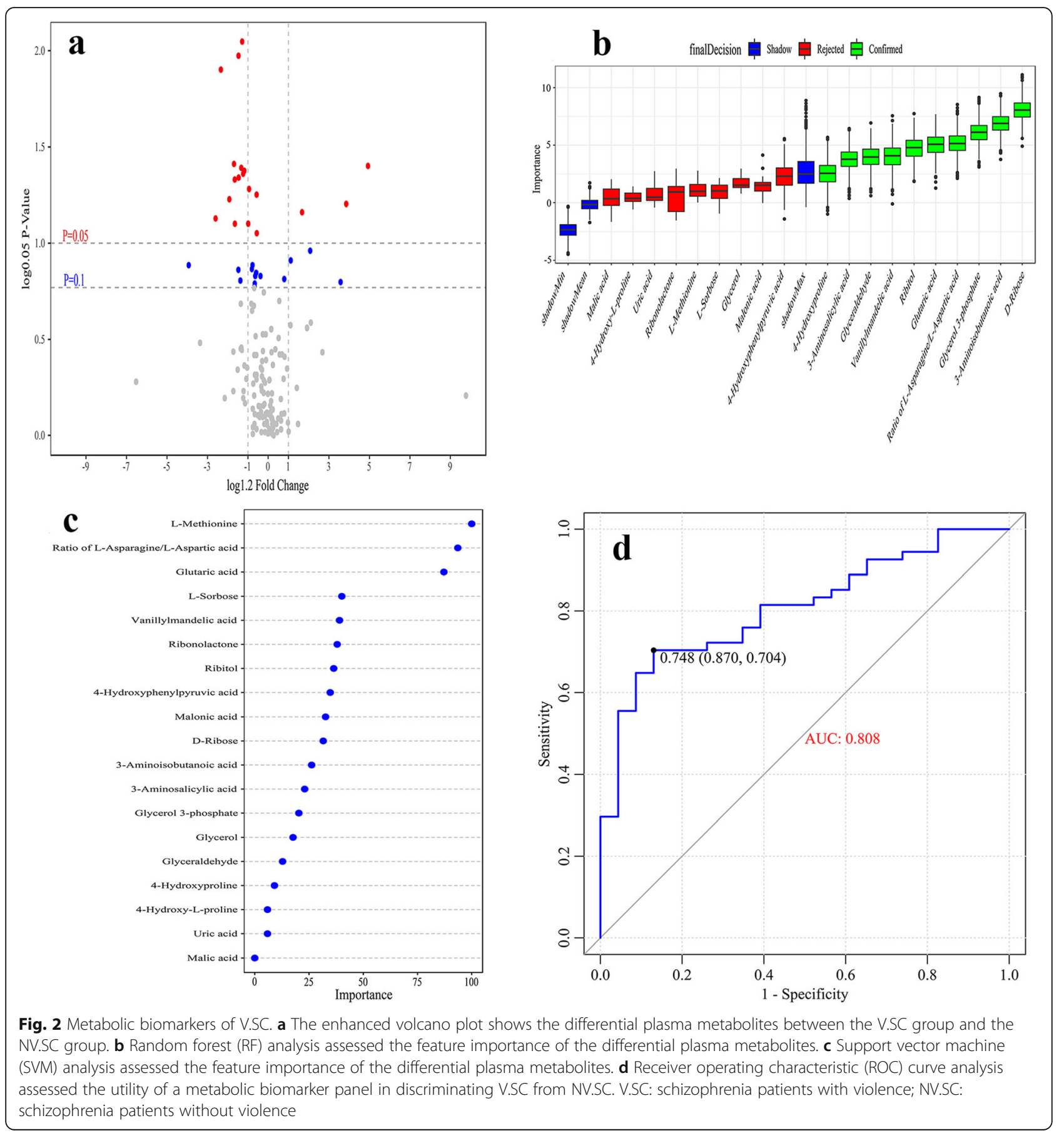

phosphate was significantly down-regulated (Fig. 3b). In the phenylalanine, tyrosine and tryptophan biosynthesis pathway, 4-hydroxyphenylpyruvic acid was significantly down-regulated (Fig. 3c).

\section{Discussion}

After controlling for confounding factors, we profiled metabolic status and identified 19 differential metabolites in the plasma of V.SC by comparing with
NV.SC. The biomarker panel, including the ratio of $\mathrm{L}$-asparagine to $\mathrm{L}$-aspartic acid, vanillylmandelic acid and glutaric acid, achieved a good classifier for discriminating schizophrenia patients with violence $(\mathrm{AUC}=0.808)$. This study detected metabolite status in schizophrenia patients with violence and found metabolic biomarkers of schizophrenia with violence. Our results can not only aid in understanding the aetiology of violence, but also suggest the plasma 
Table 2 Differential plasma metabolites selected by WilcoxonMann-Whitney rank sum test between the V.SC and NV.SC groups

\begin{tabular}{|c|c|c|c|}
\hline Pathway & Metabolites & FC & $P$ value \\
\hline \multirow[t]{6}{*}{ Amino acid metabolism } & Vanillylmandelic acid & 2.46 & 0.02 \\
\hline & Malic acid & 0.90 & 0.04 \\
\hline & 4-Hydroxyphenylpyruvic acid & 0.80 & 0.02 \\
\hline & 4-Hydroxy-L-proline & 0.84 & 0.04 \\
\hline & L-Methionine & 0.79 & 0.002 \\
\hline & $\begin{array}{l}\text { Ratio of L-asparagine/ } \\
\text { L-aspartic acid }\end{array}$ & 0.77 & 0.003 \\
\hline \multirow[t]{4}{*}{ Lipid metabolism } & Malonic acid & 0.77 & 0.02 \\
\hline & Glycerol & 2.02 & 0.03 \\
\hline & Glycerol 3-phosphate & 0.71 & 0.03 \\
\hline & Glyceraldehyde & 1.36 & 0.03 \\
\hline $\begin{array}{l}\text { Pentose phosphate } \\
\text { pathway }\end{array}$ & D-Ribose & 0.74 & 0.02 \\
\hline Purine metabolism & Uric acid & 0.74 & 0.04 \\
\hline Pyrimidine metabolism & 3-Aminoisobutanoic acid & 0.84 & 0.02 \\
\hline \multirow[t]{6}{*}{ NA } & L-Sorbose & 0.74 & 0.02 \\
\hline & 3-Aminosalicylic acid & 0.90 & 0.02 \\
\hline & Glutaric acid & 0.65 & 0.00 \\
\hline & 4-Hydroxyproline & 0.62 & 0.03 \\
\hline & Ribitol & 0.81 & 0.02 \\
\hline & Ribonolactone & 0.79 & 0.02 \\
\hline
\end{tabular}

Abbreviation: FC fold change, NA not available, V.SC schizophrenia patients with violence, NV.SC schizophrenia patients without violence

metabolites could serve as a new biomarkers for predicting violence among schizophrenia patients.

Our results showed glycerolipid metabolism and related metabolites (including glycerol, glycerol 3phosphate and glyceraldehyde) differed between V.SC and NV.SC. Notably, the patients of this study, being hospital inpatients, share similar diets, living conditions and living habits, which are confounding factors of metabolites [55-57]. Although the duration of inpatient showed difference between groups, the average years of each group was above one year. This means they had shared similar environment for an enough long time, so we do not think this could contribute much to the difference of metabolites. Glycerol 3-phosphate is a phosphoric ester of glycerol that controls the flux through the glycerolipid/free fatty acid cycle, phospholipid biosynthesis and many other cellular functions [58]. We found the glycerol 3-phosphate was decreased in V.SC, which suggests a dysregulation of lipid metabolism in schizophrenia patients with violence. This founding is consistent with previous studies reporting the lower fatty acid levels might be related to violence [23, 59]. Although earlier studies have reported the serum cholesterol, a subclass of lipid [60], is uncorrelated with violence [61, 62], more recent studies have consistently found the increased violence risk is correlated with low cholesterol concentration [19, 20,63] and polyunsaturated fatty acid $[26,59]$. Omega-3 supplement can reduce violent behaviours in children [25], young men [64], schizophrenia patients [26] and adult prisoners [23]. Our research also proves the important role of lipid metabolism in regulating violence.

In this study, five amino acids decreased in V.SC compared with NV.SC, involving in nine amino acid metabolism pathways; phenylalanine, tyrosine and tryptophan biosynthesis was a differential metabolic pathway between the two groups. The dysregulation of amino acids has been consistently correlated with psychopathology $[65,66]$. Some amino acids are correlated with violence in previous studies, especially tryptophan [67], which is the amino acid precursor of 5-HT biosynthesis [13]. A study of violent criminals found the plasma content of the amino acid tryptophan and other large neutral amino acids were increased [67-69]; however, much more studies have reported decreased tryptophan is correlated with aggressive behaviours [15, 70-72]. Instead of finding the plasma tryptophan as a differential metabolite, we found phenylalanine, tyrosine and tryptophan biosynthesis was a differential metabolic pathway between the schizophrenia patients with and without violence, which support amino acids play an important role in regulating violence.. We found vanillylmandelic acid, involved in tyrosine metabolism, was increased in V.SC, which is consistent with an earlier study reporting tyrosine supplements increased aggressive behaviours in mice [17]. Limited by techniques, many previous studies reported one or several amino acids were altered among individuals with violence, based on one or another hypothesis. Our results give a holistic view of the metabolic phenotype of violence in schizophrenia. Metabolomics should be further used to explore the role of amino acids in violence in the future.

We identified L-methionine, the ratio of L-asparagine to L-aspartic acid, glutaric acid, L-sorbose and vanillylmandelic acid as predictors of violence in schizophrenia patients. A previous study reported serotonin/tryptophan "1000, antisocial behaviour and global assessment of functioning were good predictors of aggressive behaviours among inmate (AUC $=0.851$ ) [38]. Metabolic predictors always showed variability in different studies with schizophrenia patients [32, 73, 74]; however, some consistent potential biomarkers of schizophrenia have been discovered [37]. Consequently, more metabolomics studies should be conducted to discover metabolic biomarkers of violent behaviours in schizophrenia.

There are also some limitations in this study. First, the sample size was not 1:1 between the two groups. This was led by controlling confounding factors, such as diet, 


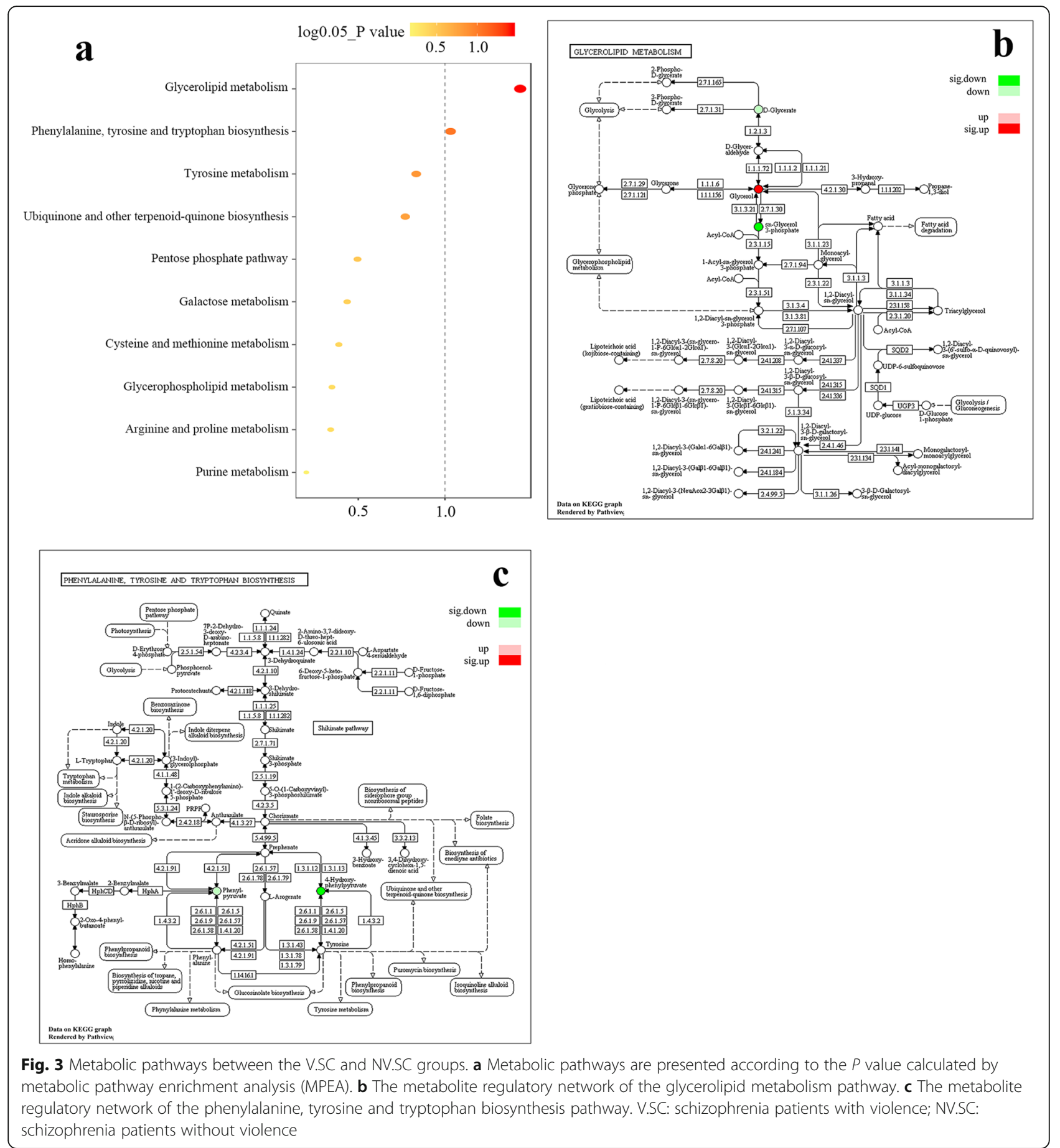

disease and so on. Second, the sample size was small and there can be over-fitting issue in classification. Small sample might be the reason of why the orthogonal partial least square discriminant analysis failed to distinguish V.SC from NV.SC. Because of controlling confounding factors of metabolites, it is difficult to collect other sample to expand sample size or validate overfitting. We used two different ways (RF and SVM) to select metabolic biomarkers from differential metabolites and chosen overlapping part of the two results (RF and SVM) to form final biomarker panel. This could not only remedy the disadvantage of small sample, but also reduce the over-fitting issue in some degree, for it might be unlikely for two different algorithms to over-fitting the same way $[48,75]$. The result of ROC analysis also validate the utility of the final biomarker panel. 
Table 3 Differential metabolic pathways identified by MPEA between the V.SC and NV.SC groups

\begin{tabular}{llll}
\hline Pathway & Up & Down & \\
\hline Glycerolipid metabolism & Glycerol & Glycerol 3-phosphate & P_her \\
Phenylalanine, tyrosine and tryptophan biosynthesis & & 4-Hydroxyphenylpyruvic acid & 0.01 \\
Tyrosine metabolism & Vanillylmandelic acid & 4-Hydroxyphenylpyruvic acid & 0.045 \\
Ubiquinone and other terpenoid-quinone biosynthesis & & 4-Hydroxyphenylpyruvic acid \\
Pentose phosphate pathway & & D-Ribose & 0.08 \\
Galactose metabolism & Glycerol & & 0.10 \\
Cysteine and methionine metabolism & & L-Methionine \\
Glycerophospholipid metabolism & & Glycerol 3-phosphate \\
Arginine and proline metabolism & & 4-Hydroxyproline & 0.27 \\
Purine metabolism & & Uric acid & 0.31
\end{tabular}

Abbreviation: MPEA metabolic pathway enrichment analysis, V.SC schizophrenia patients with violence, NV.SC schizophrenia patients without violence

However, this is a preliminary metabolomics study about schizophrenia with violence, our biomarker panel of schizophrenia with violence needs further validation in an independent sample of violent schizophrenia. Third, the length of inpatients duration is relative long. However, the minimum interval (about two months) could be enough long for the corresponding changes of plasma metabolites. Finally, the fresh blood samples were not collected immediately after the patients conducted violent behaviour, therefore the violence in this study is more inclined to be a feature than a status.

\section{Conclusions}

Our study gives a holistic view of the metabolic phenotype of violence in schizophrenia by untargeted metabolomics. We found the dysregulation of lipids and amino acids featured the metabolic phenotype of schizophrenia with violence. These results further confirm violent behaviours might be correlated with a dysregulated metabolic status, suggest that severe metabolic dysregulation might be related to violence and provide information for the aetiological understanding and management of violence in schizophrenia in the future.

\section{Supplementary information}

Supplementary information accompanies this paper at https://doi.org/10. 1186/s12888-020-02499-y.

Additional file 1. Supplementary methods and materials. Supple. Table 1. Receiver Operating Characteristic (ROC) results of differential metabolites. Supple. Figure 1. Principal components analysis (PCA) of the V.SC group and the NV.SC group.

\section{Abbreviations}

AUC: Area under curve; FC: Fold change; MPEA: Metabolic pathway enrichment analysis; NV.SC: Schizophrenia patients without violence; PANSS: Positive and Negative Syndrome Scale; RF: Random forest; ROC: Receiver operating characteristic curve; SVM: Support vector machine; V.SC: Schizophrenia patients with violence

\section{Acknowledgements}

We thank Jingcao Huang and Yi Ye, who kindly assisted with the experimental technology. We also thank Jingjie PTM BioLab (Hangzhou, China) for the sample preparation and analysis. We are also grateful to Jiaguo Luo, Gang Chen and Dan Jiang for their support in data acquisition.

\section{Authors' contributions}

Study concept and design: XCC, JMH and JJX. Data acquisition: JT, XHD, HLH and RCC. Analysis and interpretation of data: XCC and JJX. Drafting of the manuscript: XCC. Critical revision of the manuscript for important intellectual content: JMH and JJX. All authors have approved the final article.

\section{Funding}

This study was supported by the Key Research and Development Project of Science and Technology Department, Sichuan Province under Grant No. 2017SZ0062, the Graduate Student Research Innovation Foundation of Sichuan University under Grant No. 2018YJSY098.

\section{Availability of data and materials}

The datasets generated and/or analysed during the current study are not publicly available since the publication of raw data was not included in the consent forms, but all data and materials are available from the corresponding author on reasonable request.

Ethics approval and consent to participate

The ethics approval of this study was approved by the Medical Ethics Committee of Sichuan University. Legal guardians were contacted to provide written consent on behalf of the patients to participate in the study.

Consent for publication

Not applicable.

\section{Competing interests}

The authors declare that they have no competing interests.

\section{Author details}

${ }^{1}$ Institute of Forensic Medicine, West China School of Basic Medical Sciences \& Forensic Medicine, Sichuan University, Chengdu, China. ${ }^{2}$ Mental Health Center, West China Hospital, Sichuan University, Chengdu, China. ${ }^{3}$ Chengdu Compulsory Medical Center, Chengdu, China. ${ }^{4}$ West China School of Basic Medical Sciences \& Forensic Medicine, Sichuan University, Chengdu 610041, China.

Received: 10 October 2019 Accepted: 14 February 2020

Published online: 04 March 2020

\section{References}

1. Singh JP, Grann M, Lichtenstein P, Langstrom N, Fazel S. A novel approach to determining violence risk in schizophrenia: developing a stepped 
strategy in 13,806 discharged patients. PLoS One. 2012;7(2):e31727. https:// doi.org/10.1371/journal.pone.0031727.

2. Schwarz E, Maukonen J, Hyytiäinen T, Kieseppä T, Orešič M, Sabunciyan S, Mantere $\mathrm{O}$, Saarela M, Yolken R, Suvisaari J. Analysis of microbiota in first episode psychosis identifies preliminary associations with symptom severity and treatment response. Schizophr Res. 2017;192.

3. Poldrack RA, Monahan J, Imrey PB, Reyna V, Raichle ME, Faigman D, Buckholtz JW. Predicting violent behavior: what can neuroscience add? Trends Cogn Sci. 2018;22(2):111-23. https://doi.org/10.1016/j.tics.2017.11.003.

4. Brunner HG, Nelen M, Breakefield XO, Ropers HH, Van Oost BA. Abnormal behavior associated with a point mutation in the structural gene for monoamine oxidase A. Science (New York, NY). 1993;262(5133):578-80. https://doi.org/10.1126/science.8211186.

5. Tang X, Jin J, Tang Y, Cao J, Huang J. Risk assessment of aggressive behavior in Chinese patients with schizophrenia by fMRI and COMT gene. Neuropsychiatr Dis Treat. 2017;13:387-95. https://doi.org/10.2147/ndtS126356.

6. Matthies S, Rusch N, Weber M, Lieb K, Philipsen A, Tuescher O, Ebert D, Hennig J, van Elst LT. Small amygdala-high aggression? The role of the amygdala in modulating aggression in healthy subjects. World J Biol Psychiatry. 2012;13(1):75-81. https://doi.org/10.3109/15622975.2010.541282.

7. Bobes MA, Ostrosky F, Diaz K, Romero C, Borja K, Santos Y, Valdes-Sosa M. Linkage of functional and structural anomalies in the left amygdala of reactive-aggressive men. Soc Cogn Affect Neurosci. 2013;8(8):928-36. https://doi.org/10.1093/scan/nss101.

8. Rudebeck PH, Murray EA. The orbitofrontal oracle: cortical mechanisms for the prediction and evaluation of specific behavioral outcomes. Neuron. 2014;84(6):1143-56. https://doi.org/10.1016/..neuron.2014.10.049.

9. Fulwiler $\mathrm{CE}$, King JA, Zhang N. Amygdala-orbitofrontal resting-state functional connectivity is associated with trait anger. Neuroreport. 2012; 23(10):606-10. https://doi.org/10.1097/WNR.0b013e3283551cfc.

10. Fjellvang M, Groning L, Haukvik UK. Imaging violence in schizophrenia: a systematic review and critical discussion of the MRI literature. Front Psychiatry. 2018;9:333. https://doi.org/10.3389/fpsyt.2018.00333.

11. Cho W, Shin WS, An I, Bang M, Cho DY, Lee SH. Biological aspects of aggression and violence in schizophrenia. Clin Psychopharmacol Neurosci. 2019;17(4):475-86. https://doi.org/10.9758/cpn.2019.17.4.475.

12. Rosell DR, Siever $L$. The neurobiology of aggression and violence. CNS spectrums. 2015;20(3):254-79. https://doi.org/10.1017/s109285291500019x.

13. Carpenter LL, Anderson GM, Pelton GH, Gudin JA, Kirwin PD, Price LH, Heninger GR, McDougle CJ. Tryptophan depletion during continuous CSF sampling in healthy human subjects. Neuropsychopharmacology. 1998; 19(1):26-35. https://doi.org/10.1016/s0893-133x(97)00198-x.

14. Moreno FA, McGavin C, Malan TP, Gelenberg AJ, Heninger GR, Mathe AA, Delgado PL. Tryptophan depletion selectively reduces CSF 5-HT metabolites in healthy young men: results from single lumbar puncture sampling technique. Int J Neuropsychopharmacol. 2000;3(4):277-83. https://doi.org/ 10.1017/s1461145700002133.

15. Bjork JM, Dougherty DM, Moeller FG, Swann AC. Differential behavioral effects of plasma tryptophan depletion and loading in aggressive and nonaggressive men. Neuropsychopharmacology. 2000;22(4):357-69. https:// doi.org/10.1016/s0893-133x(99)00136-0.

16. von Polier GG, Biskup CS, Kotting WF, Bubenzer S, Helmbold K, Eisert A, Gaber TJ, Zepf FD. Change in electrodermal activity after acute tryptophan depletion associated with aggression in young people with attention deficit hyperactivity disorder (ADHD). J Neural Transm (Vienna). 2014;121(4):451-5. https://doi.org/10.1007/s00702-013-1119-5.

17. Thurmond JB, Lasley SM, Kramarcy NR, Brown JW. Differential tolerance to dietary amino acid-induced changes in aggressive behavior and locomotor activity in mice. Psychopharmacology. 1979;66(3):301-8. https://doi.org/10. 1007/bf00428324'

18. Pliashkevich lu G, Barsegian GG, Ponomarev S, Demushkin VP. Effect of amino acid derivatives of beta-carboline-3-carboxylate on rat behavior. Biull Eksp Biol Med. 1989;107(5):586-7.

19. Tomson-Johanson K, Harro J. Low cholesterol, impulsivity and violence revisited. Curr Opin Endocrinol Diabetes Obes. 2018;25(2):103-7. https://doi. org/10.1097/med.00000000000000395.

20. Kraav SL, Tolmunen T, Karkkainen O, Ruusunen A, Viinamaki H, Mantyselka P, Koivumaa-Honkanen $\mathrm{H}$, Valkonen-Korhonen M, Honkalampi K, Herzig KH, et al. Decreased serum total cholesterol is associated with a history of childhood physical violence in depressed outpatients. Psychiatry Res. 2019; 272:326-33. https://doi.org/10.1016/j.psychres.2018.12.108.
21. Eriksen BMS, Bjorkly S, Lockertsen O, Faerden A, Roaldset JO. Low cholesterol level as a risk marker of inpatient and post-discharge violence in acute psychiatry - a prospective study with a focus on gender differences. Psychiatry Res. 2017;255:1-7. https://doi.org/10.1016/j.psychres.2017.05.010.

22. Chen SC, Chu NH, Hwu HG, Chen WJ. Trajectory classes of violent behavior and their relationship to lipid levels in schizophrenia inpatients. J Psychiatr Res. 2015;66-67:105-11. https://doi.org/10.1016/j.jpsychires.2015.04.022.

23. Gesch CB, Hammond SM, Hampson SE, Eves A, Crowder MJ. Influence of supplementary vitamins, minerals and essential fatty acids on the antisocial behaviour of young adult prisoners. Randomised, placebo-controlled trial. $\mathrm{Br}$ J Psychiatry. 2002;181:22-8. https://doi.org/10.1192/bjp.181.1.22.

24. Sagduyu K, Dokucu ME, Eddy BA, Craigen G, Baldassano CF, Yildiz A. Omega-3 fatty acids decreased irritability of patients with bipolar disorder in an add-on, open label study. Nutr J. 2005;4:6. https://doi.org/10.1186/1475-2891-4-6.

25. Raine A, Portnoy J, Liu J, Mahoomed T, Hibbeln JR. Reduction in behavior problems with omega-3 supplementation in children aged 8-16 years: a randomized, double-blind, placebo-controlled, stratified, parallel-group trial. J Child Psychol Psychiatry Allied Disciplines. 2015;56(5):509-20. https://doi. org/10.1111/jcpp.12314

26. Qiao Y, Mei Y, Han H, Liu F, Yang XM, Shao Y, Xie B, Long B. Effects of Omega-3 in the treatment of violent schizophrenia patients. Schizophr Res. 2018;195:283-5. https://doi.org/10.1016/j.schres.2017.08.026.

27. Portnoy J, Raine A, Liu J, Hibbeln JR. Reductions of intimate partner violence resulting from supplementing children with omega-3 fatty acids: a randomized, double-blind, placebo-controlled, stratified, parallel-group trial. Aggress Behav. 2018. https://doi.org/10.1002/ab.21769.

28. Gajos JM, Beaver KM. The effect of omega-3 fatty acids on aggression: a meta-analysis. Neurosci Biobehav Rev. 2016;69:147-58. https://doi.org/10. 1016/j.neubiorev.2016.07.017.

29. Annalaura M, Armitage EG, Antonia G, Coral B. Metabolomics as a tool for drug discovery and personalised medicine. A review. Curr Top Med Chem. 2014;14(23):2627-36

30. Wishart DS. Emerging applications of metabolomics in drug discovery and precision medicine. Nat Rev Drug Discov. 2016;15(7):473-84.

31. Liu ML, Zheng P, Liu Z, Xu Y, Mu J, Guo J, Huang T, Meng HQ, Xie P. GC-MS based metabolomics identification of possible novel biomarkers for schizophrenia in peripheral blood mononuclear cells. Mol BioSyst. 2014; 10(9):2398-406.

32. Yang J, Chen T, Sun L, Zhao Z, Qi X, Zhou K, Cao Y, Wang X, Qiu Y, Su M, et al. Potential metabolite markers of schizophrenia. Mol Psychiatry. 2013; 18(1):67-78. https://doi.org/10.1038/mp.2011.131.

33. Koike S, Bundo M, Iwamoto K, Suga M, Kuwabara H, Ohashi Y, Shinoda K, Takano Y, Iwashiro N, Satomura Y. A snapshot of plasma metabolites in firstepisode schizophrenia: a capillary electrophoresis time-of-flight mass spectrometry study. Transl Psychiatry. 2014;4(4):e379.

34. Cai H-L, Li H-D, Yan X-Z, Sun B, Zhang Q, Yan M, Zhang W-Y, Jiang P, Zhu R$\mathrm{H}$, Liu Y-P, et al. Metabolomic analysis of biochemical changes in the plasma and urine of first-episode neuroleptic-Naïve schizophrenia patients after treatment with Risperidone. J Proteome Res. 2012;11(8):4338-50. https://doi.org/10.1021/pr300459d.

35. Pawelczyk T, Grancow M, Kotlicka-Antczak M, Trafalska E, Gebski P, Szemraj J, Zurner N, Pawelczyk A. Omega-3 fatty acids in first-episode schizophrenia a randomized controlled study of efficacy and relapse prevention (OFFER): rationale, design, and methods. BMC Psychiatry. 2015;15:97. https://doi.org/ 10.1186/s12888-015-0473-2.

36. Horrobin DF, Glen AIM, Vaddadi K. The membrane hypothesis of schizophrenia. Schizophr Res. 1994;13(3):195-207. https://doi.org/10.1016/ 0920-9964(94)90043-4.

37. Davison J, O'Gorman A, Brennan L, Cotter DR. A systematic review of metabolite biomarkers of schizophrenia. Schizophr Res. 2018;195:32-50. https://doi.org/10.1016/j.schres.2017.09.021.

38. Comai S, Bertazzo A, Vachon J, Daigle M, Toupin J, Cote G, Turecki G, Gobbi G. Tryptophan via serotonin/kynurenine pathways abnormalities in a large cohort of aggressive inmates: markers for aggression. Prog NeuroPsychopharmacol Biol Psychiatry. 2016;70:8-16. https://doi.org/10.1016/j. pnpbp.2016.04.012.

39. Kuehnbaum NL, Philip BM. New advances in separation science for metabolomics: resolving chemical diversity in a post-genomic era. Chem Rev. 2013;113(4):2437-68.

40. Hagenbeek FA, Kluft C, Hankemeier T, Bartels M, Draisma HHM, Middeldorp CM, Berger R, Noto A, Lussu M, Pool R, et al. Discovery of biochemical 
biomarkers for aggression: a role for metabolomics in psychiatry. Am J Med Genet B Neuropsychiatr Genet. 2016;171(5):719-32. https://doi.org/10.1002/ ajmg.b.32435

41. Collins SM, Surette M, Bercik P. The interplay between the intestinal microbiota and the brain. Nat Rev Microbiol. 2012;10(11):735-42. https://doi. org/10.1038/nrmicro2876.

42. Rohrscheib CE, Bondy E, Josh P, Riegler M, Eyles D, van Swinderen B, Weible MW, Brownlie JC. Wolbachia influences the production of Octopamine and affects Drosophila male aggression. Appl Environ Microbiol. 2015;81(14): 4573.

43. Monahan J, Steadman HJ, Silver E, Appelbaum PS, Clark Robbins P. Mulvey EP Ea. rethinking risk assessment. The MacArthur study of mental disorder and violence. New York: NY: Oxford University Press; 2001.

44. Cheng Y, Xie G, Chen T, Qiu Y, Zou X, Zheng M, Tan B, Feng B, Dong T, He $P$, et al. Distinct urinary metabolic profile of human colorectal cancer. J Proteome Res. 2012;11(2):1354-63. https://doi.org/10.1021/pr201001a.

45. Pinto RC. Chemometrics methods and strategies in metabolomics. Adv Exp Med Biol. 2017;965:163-90. https://doi.org/10.1007/978-3-319-47656-8_7.

46. Blasco H, Blaszczynski J, Billaut JC, Nadal-Desbarats L, Pradat PF, Devos D, Moreau C, Andres CR, Emond P, Corcia P, et al. Comparative analysis of targeted metabolomics: dominance-based rough set approach versus orthogonal partial least square-discriminant analysis. J Biomed Inform. 2015; 53:291-9. https://doi.org/10.1016/j.jbi.2014.12.001.

47. Boccard J, Rutledge DN. A consensus orthogonal partial least squares discriminant analysis (OPLS-DA) strategy for multiblock Omics data fusion. Anal Chim Acta. 2013;769:30-9. https://doi.org/10.1016/j.aca.2013.01.022.

48. Kursa MB, Rudnicki WR. Feature selection with the boruta package. J Stat Softw. 2010;36(11):1-13. https://doi.org/10.18637/jss.v036.i11.

49. Lin X, Wang Q, Yin P, Tang L, Tan Y, Li H, Yan K, Xu G. A method for handling metabonomics data from liquid chromatography/mass spectrometry: combinational use of support vector machine recursive feature elimination, genetic algorithm and random forest for feature selection. Metabolomics. 2011;7(4):549-58. https://doi.org/10.1007/s11306011-0274-7.

50. Heinemann J, Mazurie A, Tokmina-Lukaszewska M, Beilman GJ, Bothner B. Application of support vector machines to metabolomics experiments with limited replicates. Metabolomics. 2014;10(6):1121-8. https://doi.org/10.1007/ s11306-014-0651-0.

51. Erban A, Fehrle I, Martinez-Seidel F, Brigante F, Más AL, Baroni V, Wunderlin D, Kopka J. Discovery of food identity markers by metabolomics and machine learning technology. Sci Rep. 2019;9(1):9697. https://doi.org/10. 1038/s41598-019-46113-y.

52. Guan W, Zhou M, Hampton CY, Benigno BB, Walker LD, Gray A, McDonald JF, Fernandez FM. Ovarian cancer detection from metabolomic liquid chromatography/mass spectrometry data by support vector machines. BMC Bioinformatics. 2009;10:259. https://doi.org/10.1186/1471-2105-10-259.

53. Kuhn M. Building predictive models in $\mathrm{R}$ using the caret package. J Stat Softw 2008.

54. Kankainen M, Gopalacharyulu P, Holm L, Oresic M. MPEA--metabolite pathway enrichment analysis. Bioinformatics (Oxford, England). 2011;27(13): 1878-9. https://doi.org/10.1093/bioinformatics/btr278.

55. Sussulini A. Metabolomics: from fundamentals to clinical applications. Switzerland: Springer Nature; 2017.

56. Thévenot EA, Roux A, Xu Y, Ezan E, Junot $C$. Analysis of the human adult urinary Metabolome variations with age, body mass index, and gender by implementing a comprehensive workflow for Univariate and OPLS statistical analyses. J Proteome Res. 2015;14(8):3322-35. https://doi.org/10.1021/acs. jproteome.5b00354.

57. Dunn WB, Lin W, Broadhurst D, Begley P, Brown M, Zelena E, Vaughan AA Halsall A, Harding N, Knowles JD, et al. Molecular phenotyping of a UK population: defining the human serum metabolome. Metabolomics. 2015; 11:9-26. https://doi.org/10.1007/s11306-014-0707-1.

58. Prentki M, Madiraju SR. Glycerolipid metabolism and signaling in health and disease. Endocr Rev. 2008;29(6):647-76. https://doi.org/10.1210/er.2008-0007.

59. Buydens-Branchey L, Branchey M, McMakin DL, Hibbeln JR. Polyunsaturated fatty acid status and aggression in cocaine addicts. Drug Alcohol Depend. 2003;71(3):319-23. https://doi.org/10.1016/S0376-8716(03)00168-6.

60. Sethi S, Hayashi MA, Sussulini A, Tasic L, Brietzke E. Analytical approaches for lipidomics and its potential applications in neuropsychiatric disorders. World J Biol Psychiatry. 2017;18(7):506-20. https://doi.org/10.3109/15622975.2015. 1117656.
61. Huang T, Wu S. Serum cholesterol levels in paranoid and non-paranoid schizophrenia associated with physical violence or suicide attempts in Taiwanese. Psychiatry Res. 2000;96(2):175-8.

62. Steinert T, Woelfle M, Gebhardt RP. No correlation of serum cholesterol levels with measures of violence in patients with schizophrenia and nonpsychotic disorders. Eur Psychiatry. 1999;14(6):346-8.

63. Asellus P, Nordstrom P, Nordstrom AL, Jokinen J. Cholesterol and the "cycle of violence" in attempted suicide. Psychiatry Res. 2014;215(3):646-50. https://doi.org/10.1016/j.psychres.2014.01.009.

64. Long SJ, Benton D. A double-blind trial of the effect of docosahexaenoic acid and vitamin and mineral supplementation on aggression, impulsivity, and stress. Hum Psychopharmacol. 2013;28(3):238-47. https://doi.org/10. 1002/hup.2313.

65. Ruppel RA, Kochanek PM, Adelson PD, Rose ME, Wisniewski SR, Bell MJ, Clark RS, Marion DW, Graham SH. Excitatory amino acid concentrations in ventricular cerebrospinal fluid after severe traumatic brain injury in infants and children: the role of child abuse. J Pediatr. 2001;138(1):18-25. https:// doi.org/10.1067/mpd.2001.110979.

66. Smith AM, King JJ, West PR, Ludwig MA, Donley ELR, Burrier RE, Amaral DG. Amino acid Dysregulation Metabotypes: potential biomarkers for diagnosis and individualized treatment for subtypes of autism Spectrum disorder. Biol Psychiatry. 2019;85(4):345-54. https://doi.org/10.1016/j.biopsych.2018.08.016.

67. Eriksson T, Lidberg L. Increased plasma concentrations of the $5-H T$ precursor amino acid tryptophan and other large neutral amino acids in violent criminals. Psychol Med. 1997;27(2):477-81.

68. Vignau J, Soichot M, Imbenotte M, Jacquemont MC, Danel T, Vandamme M, Lhermitte M, Allorge D. Impact of tryptophan metabolism on the vulnerability to alcohol-related blackouts and violent impulsive behaviours. Alcohol Alcohol (Oxford, Oxfordshire). 2010;45(1):79-88. https://doi.org/10. 1093/alcalc/agp044.

69. Tiihonen J, Virkkunen M, Räsänen P, Pennanen S, Sainio EL, Callaway J, Halonen $P$, Liesivuori J. Free $L$-tryptophan plasma levels in antisocial violent offenders. Psychopharmacology. 2001;157(4):395-400.

70. Bjork JM, Dougherty DM, Moeller FG, Cherek DR, Swann AC. The effects of tryptophan depletion and loading on laboratory aggression in men: time course and a food-restricted control. Psychopharmacology. 1999;142(1):2430. https://doi.org/10.1007/s002130050858.

71. Zimmermann M, Grabemann M, Mette C, Abdel-Hamid M, Uekermann J, Kraemer M, Wiltfang J, Kis B, Zepf FD. The effects of acute tryptophan depletion on reactive aggression in adults with attention-deficit/ hyperactivity disorder (ADHD) and healthy controls. PLoS One. 2012;7(3): e32023. https://doi.org/10.1371/journal.pone.0032023.

72. Kotting WF, Bubenzer S, Helmbold K, Eisert A, Gaber TJ, Zepf FD. Effects of tryptophan depletion on reactive aggression and aggressive decisionmaking in young people with ADHD. Acta Psychiatr Scand. 2013;128(2):11423. https://doi.org/10.1111/acps.12001.

73. Fukushima T, lizuka H, Yokota A, Suzuki T, Ohno C, Kono Y, Nishikiori M, Seki A, Ichiba $H$, Watanabe $Y$. Quantitative analyses of schizophrenia-associated metabolites in serum: serum D-lactate levels are negatively correlated with gamma-Glutamylcysteine in medicated schizophrenia patients. PLoS One 2014;9(7):e101652

74. Ballesteros A, Summerfelt A, Du X, Jiang P, Chiappelli J, Tagamets M, O'Donnell P, Kochunov P, Hong LE. Electrophysiological intermediate biomarkers for oxidative stress in schizophrenia. Clin Neurophysiols. 2013; 124(11):2209-15. https://doi.org/10.1016/j.clinph.2013.05.021.

75. Rojarath A, Songpan W, Pong-inwong C. Improved ensemble learning for classification techniques based on majority voting. In: 2016 7th IEEE international conference on software engineering and service science (ICSESS). Beijing, China: IEEE; 2016. p. 107-10.

\section{Publisher's Note}

Springer Nature remains neutral with regard to jurisdictional claims in published maps and institutional affiliations. 